\title{
RELEYENDO LA JURISPRUDENCIA NORMATIVA DE RONALD DWORKIN: UNA VALORACIÓN DE CONJUNTO*
}

\author{
Giuseppe Zaccaria \\ Universidad de Padua
}

RESUMEN: En este trabajo se evalúa el impacto sobre la filosofía del derecho contemporánea de la obra de Ronald DWORKIN. A partir de HART y en lo sucesivo, no se ha podido dejar de debatir las ideas de DWORKIN y de dedicarles ensayos y conferencias. Debe reconocerse que DwORKIN ha cambiado profundamente el panorama de la teoría del derecho contemporánea, contribuyendo de manera decisiva a abandonar definitivamente la concepción vetero-positivista del derecho y reorientando completa y profundamente el debate sobre el positivismo jurídico y sobre el iusnaturalismo.

Palabras clave: DwORKIN, positivismo jurídico, iusnaturalismo, interpretación, argumentación moral.

\section{Re-reading normative jurisprudence of Ronald Dworkin. A global valuation}

ABSTRACT: This paper examines the impact of Ronald DwORKIN's work on contemporary Legal Philosophy. Ever since HART, DwORKIN's ideas have been constantly on debate. Many essays and lectures have been devoted to them. DwORKIN has deeply changed contemporary Legal Theory landscape, contributing enormously to definitely abandoning the classic-positivist conception of Law and fully and deeply reorienting the debate on Legal Positivism and Natural Law Theories. soning.

Keywords: DwORKIN, legal positivism, Natural Law Theories, interpretation, moral rea-

* Fecha de recepción: 18 de septiembre de 2015. Fecha de aceptación: 9 de diciembre de 2015.

Traducción de Gregorio ROBLES MORCHÓN. 


\section{LA OBRA DE RONALD DWORKIN, UNA MINA DE ARGUMENTOS PARA EL FILÓSOFO DEL DERECHO}

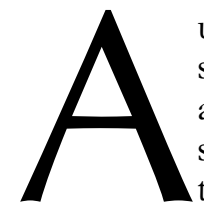

un año de la desaparición de Ronald DwORKIN (1931-2013), un estudioso sobre cuya obra existe ya una literatura muy abundante, se percibe como algo ya ineludible una reflexión orgánica y no contingente sobre su pensamiento. Ronald DwORKIN, de hecho, no es solo uno entre los filósofos y teóricos del derecho de mayor relevancia en el plano internacional, en los últimos años del siglo XX y en la primera década de siglo XXI, también representa una de las voces más autorizadas dentro de un amplísimo arco de reflexiones que han analizado puntos cruciales y clásicos de la filosofía no solo jurídica sino también moral y política, así como la relación entre reglas y principios, la existencia de discrecionalidad del juez y su papel en el contexto constitucional, la relación entre igualdad y libertad social, la fundamentación ontológica de las reglas, el fundamento de los valores y su realdad objetiva, el valor central de la dignidad de la vida.

Desde el fulgurante comienzo de su rights theory, en 1977, con Taking Rigths Seriously — verdadero desafío a la tesis del positivismo jurídico sobre la existencia o no de una respuesta justa en los casos jurídicos complejos- a la etapa sucesiva de $A$ Mater of Principle del 1985, lugar de elaboración de una teoría de la igualdad liberal, desde la admirable síntesis de Law's Empire del 1986 a la reflexión sobre los bienes primarios y sobre la fundamentación de los derechos fundamentales sobre la dignidad humana de Freedom's Law de 1986 y Sovering Virtue (publicado en el 2000), a través del debate sobre aspectos críticos de la democracia y sobre los modos para su posible superación a la luz de la distinción entre democracia mayoritaria y democracia participativa (Is Democracy Possible Here?, 2006), hasta los últimos Justice in Robes del 2006, sobre la intersección entre casos jurisprudenciales y cuestiones morales, Justice for Hedgehogs (del 2011) sobre la problemática fundamental de la unidad del valor, $y$ Religion without God, póstumo, sobre el papel de la religión en la política. El conjunto de sus escritos constituye una riquísima mina de análisis, desarrollados de manera brillante, con claridad ejemplar, con notable capacidad analítica y argumentativa y con constante voluntad de diálogo con otras posiciones importantes del pensamiento filosóficojurídico y filosófico-político contemporáneo. Y, sin embargo, aunque fue sin duda uno de los más grandes filósofos del derecho y de la política, DwORKIN fue también uno de los autores más discutidos y controvertidos, como demuestra el amplísimo debate, no exento de críticas despiadadas, que siguió a su audaz ataque teórico anti-positivista a la jurisprudence dominante de HART.

Aquí hay indudablemente un núcleo central en el elegante y amplísimo panorama intelectual que liga, de modo admirable, los ámbitos del derecho, de la moral y de la política y de lo que pocos, incluso entre los críticos más feroces, no se han podido resistir a la fascinación: y es sobre esto sobre lo que pretendemos concentrar nuestra atención crítica. Este núcleo está representado por la jurisprudence normativa de Ronald DwORKIN, por su interpretivist theory of law, caracterizada, al menos, por dos aspectos de fondo: la tesis de la interconexión inescindible entre derecho y moral, y la de la estrechísima relación entre las posiciones teóricas discutidas y el profundo examen de casos judiciales concretos a propósito de los que emergen, y son analizadas, discrepan- 
cias teóricas. Por consiguiente es sobre la jurisprudence de DwORKIN, y en particular sobre su giro interpretativo, donde nos detendremos en esta sintética relectura de la obra de investigación de un estudioso, que aunque desaparecido hace muy poco, y aunque pueda parecer en algunos aspectos como «un solitario tanto en el mundillo de los juristas como en el de los filósofos» ${ }^{1}$, ya de inmediato a su desaparición se le puede sin duda calificar como un verdadero clásico del pensamiento jurídico contemporáneo.

\section{LA CRÍTICA ANTI- POSITIVISTA AL MODELO HARTIANO}

El inicio de la reflexión jurídica de DwORKIN, con su primera gran obra Taking Rights Seriously ${ }^{2}$ se produce con una original y clamorosa crítica a Herbert HART y a su tesis fundamental que define el derecho como un sistema de normas. El modelo de HART, plasmado en The Concept of Law del $1961^{3}$, con su fundamental distinción entre normas primarias (que atribuyen derechos e imponen obligaciones) y normas secundarias (que establecen cómo y por quién deben de ser emanadas las reglas primarias) y su concepción de la norma de reconocimiento como criterio de validez y de identificabilidad de las normas jurídicas, gozaba, en la segunda mitad de los años setenta del siglo pasado, de indiscutida autoridad en el milieu del positivismo jurídico contemporáneo, en particular en la variante que de él ofrece la filosofía analítica, que pretendía inteligentemente salvarse de las simplificaciones de AUSTIN. La audacia de la crítica a HART estaba precisamente en asumir una completa concepción del derecho, la dominante en el ámbito anglosajón, que encontraba en la obra maestra de HART su núcleo teórico indiscutido.

DWORKIN se convertiría a continuación en el sucesor de HART en la prestigiosa cátedra de Oxford y habría vuelto a confirmar si bien mucho más tarde su posición (DwORKIN, 2010a: 5), rechazando las críticas contenidas en el Postcript de HART (en la segunda edición de The Concept of Law ${ }^{4}$ y reafirmando con vigor el «genio y la pasión» de Herbert HART (DWORKIN, 2010b: 203) pero también su firme posición de distanciamiento de las limitaciones de su jurisprudence analítica. Sin embargo en aquel momento, con la atrevida crítica a The Concept of Law -el más influyente e importante ensayo en la tradición analítica de la jurisprudence de la segunda mitad del siglo XXprovocó un gran impacto ya que resquebrajaba e introducía una brecha imprevista en la ordenada arquitectura hartiana y encendía una chispa destinada a revolucionar la teoría jurídica contemporánea. La tesis era muy sencilla pero al mismo tiempo radical, al querer archivar la antigua pero cada vez más estéril contraposición entre iusnaturalismo y positivismo jurídico: concebir el derecho como constituido exclusivamente por normas, cuyo conjunto agotaría por completo el derecho, y consecuentemente sostener que son las reglas las que establecen las fuentes autorizadas para resolver la cuestión del derecho aplicable, tesis que deja en la sombra el importante papel desarrollado por los

${ }^{1}$ La definición es de Habermas (2011: 156). Del difícil encuadre en el pensamiento de DwORKIN habla también Mackie (1983). Cfr. También RIPSTEIN (2007). El mismo DwORKIN nos ofrece una mirada lúcida y sintética sobre sus escritos precedentes (2013b).

2 Sobre esta obra hay que consultar la nota crítica de RAZ (1978).

3 Sobre esta obra clásica de HART, $c f r$. por último, DuARTE D’Almeida, EdWARDS y DOLCetTi (2013).

4 Sobre el Postscript, cfr. Coleman (2001). La tesis central y más relevante del Apéndice de HaRT y discutida por DwORKIN (2010b: 153-203). Sobre esta obra de DwORKIN hay que ver la nota de GREEN (2007). 
principios, en cuanto normas no equiparables a reglas, y más en general la relevancia de la interpretación en las decisiones judiciales: aspectos, estos, que son sin embargo esenciales si se les coloca dentro de un cuadro en el que moral y derecho, aun permaneciendo como sistemas independientes entre ellos, sin embargo presentan relaciones y conexiones profundas ${ }^{5}$. Esta tesis de una «fuerte» distinción entre reglas y principios ${ }^{6}$, sobre la base de que los principios representan los valores supremos del ordenamiento ${ }^{7}$, pueden originar diversos cursos de acciones, pueden entrar en conflicto entre ellos y tener un peso distinto y por consiguiente requieren ponderación y equilibrio para ser aplicados ${ }^{8}$, y son finalmente caracterizados por su mayor carácter genérico e indeterminado (DWORKIN, 2010a: cap. 2; cfr. ENDICOTT, 2013), tanto del supuesto de hecho como de las consecuencias jurídicas, precisamente por redefinir a fondo la estructura del derecho, lo que originaba una profunda refutación de uno de los asuntos teóricos del iuspositivismo, aquel por el que las normas representan las únicas directrices que vinculan de manera enérgica a los jueces. Al contrario, en la idea de DwORKIN con referencia a supuestos de hecho específicos hay siempre más de un principio relevante y por tanto la jerarquía axiológica es móvil en los casos reales. A su vez los principios venían, en aquella perspectiva iuspositivista, rebajados al rango de meros estándares extrajurídicos, que no determinaban resultados jurídicamente apreciables, y que pueden ser elegidos o no por el juez en plena discrecionalidad. Las teorías iuspositivistas para las que no existen obligaciones jurídicas que no sean generadas por normas (la llamada obligation thesis) ignoran los muchos casos en los que los jueces se consideran vinculados por el derecho incluso si normas precisas no se declaran aplicables. A este propósito, sin embargo, la posición de DwORKIN sufre una clara evolución, ya que en un primer momento él niega al juez la posibilidad de recurrir a estándares extralegales para resolver las controversias de su competencia, mientras que en una segunda fase reconoce que una norma social impone una obligación vinculante si, y solo si, los componentes del grupo están de acuerdo sobre el hecho de que la obligación debe de ser cumplida. Pero, en el fondo, el verdadero núcleo de controversia con HART está en la circunstancia de que deben ser considerados vinculantes en razón de su contenido moral y no por el hecho de que estos sean socialmente designados como vinculantes en base a la llamada pedigree thesis.

Su concepción del Law as integrity, entendida como alternativa indirecta a la idea de norma de reconocimiento de HART, en cuanto capaz de incluir «un conjunto de estándares mutables, interconectados y en evolución» (DWORKIN, 2010a: 73), presenta en cambio la novedad de asumir el punto de vista de la moralidad política dentro de una amplia visión del derecho, por la que la misma teoría política se basa sobre juicios y convicciones de carácter moral, e incluye entre sus criterios el carácter correcto de la solución desde un punto de vista ético-político. En el momento de resolver las con-

5 El límite para mantener en Taking Rigths Seriusly la separación entre los dos sistemas de la moral y del derecho lo reconocería muchos años después el mismo DwORKIN (2013a: 456 y ss.) reconociendo una muy estrecha conexión entre derecho y moral y llegando a identificar y a tratar el derecho como una parte de la política.

${ }^{6}$ La tesis de DwORKIN (2010a) vendrá luego retomada por autores como AleXY (2012: cap. 3; 2000); AtienZa y RuIz MANERo (1996). En la literatura italiana el tema de principios, cfr. PINTORE (1982); PASTORE (1985); ZAGREBELSKY (1992: cap. 6); MENGONi (1996); ZACCARIA (1996: 197-246); COMANDUCCI (1997); PiNO (2010: 51-75).

7 ATIENZA y Ruiz MANERo (1993).

8 Este aspecto de los principios ha sido subrayado por ALEXY (1997: 71 y ss.). 
troversias —esta es la tesis de DwORKIN (129-196) en los ensayos de finales de los años setenta sobre la solución correcta para casos difíciles ${ }^{9}$ - el juez debe de identificar el derecho existente sobre la base de los principios generales del derecho y actuando como órgano de una «comunidad personificada» que expresa una concepción de equidad y justicia. La respuesta correcta debe de ser justa según una doctrina ético-política general. Tesis, estas, como se puede fácilmente advertir, notablemente heterodoxas respecto a la separation thesis iuspositivista y respecto a la concepción de la legalidad expresada por los iuspositivistas, por la cual la moral no resulta pertinente para la definición del derecho.

Hasta este momento, sin embargo, aun distanciándose sensible y originalmente de la ortodoxia analítica - tanto que Neil MACCORMICK tuvo la oportunidad de denunciar el carácter contradictorio entre la tesis de la solución correcta y las posiciones de un construccionismo epistemológico riguroso, la perspectiva de DwORKIN, aunque muy centrada en las temáticas de la interpretación, y en particular en la interpretación literaria como posible paradigma para la interpretación jurídica (ZACCARIA, 1996: 205) ${ }^{10}$, se manifiesta todavía muy extraña al núcleo más auténtico de la hermenéutica filosófica europea. Es verdad que el problema del carácter correcto de la respuesta del intérprete se plantea dentro del contexto de una determinada práctica social y, por consiguiente, en un modo que permite construir un modelo más realista en lo que se refiere a la complejidad de las prácticas interpretativas, pero es también cierto que la teoría hermenéutica postheideriana (desde GADAMER a RICOEUR hasta PEREYSON) jamás habría suscrito la idea de DwORKIN de una y solo una solución correcta. Incluso luchando contra el malentendido, esta admite la posibilidad de una pluralidad de respuestas sostenibles y correctas. Por consiguiente el primerísimo DwORKIN, el de la única respuesta correcta. Incluso luchando contra el malentendido, admite la posibilidad de una pluralidad de respuestas sostenibles y correctas. Por consiguiente, el primerísimo DwORKIN, el de la única respuesta correcta, muestra toda su extrañeza frente al núcleo más auténtico de la hermenéutica europeo-continental y su originaria descendencia, aun en un diálogo cada vez más crítico, del modelo hartiano de interpretación jurídica: pero como él mismo reconocerá cuarenta años más tarde en Justice for Hedgehogs, concebía y defendía sus tesis sobre la interpretación dentro de un cuadro complejo que consideraba todavía derecho y moral como dos sistemas independientes entre ellos.

\section{EL «INTERPRETIVISMO» DE DWORKIN}

Donde en cambio los relevantes intereses hermenéuticos de DwORKIN comienzan a acercarse y a unirse explícitamente, aunque sea por caminos diversos, a los resultados de la perspectiva hermenéutica continental, es en la segunda fase de su reflexión sobre el derecho y más precisamente en la concepción contenida en $A$ Mater of Principle, del derecho como «exercise in interpretation» (DWORKIN, 1990b: 179) y todavía más en Law's Empire ${ }^{11}$, donde se reconoce el carácter inventivo de la actividad interpretativa

\footnotetext{
9 Cfr. ZACCARIA (1996: 205 y ss.).

10 Sobre la perspectiva del derecho como literatura, $c f r$. Minda (2001: 249 y ss.).

11 Sobre esta obra, central en la producción de DwORKIN, cfr. HeRSHOVITZ (2006).
} 
(DWORKIN 1989: 215), y se concibe al interprete como aquel que es llamado a superar una distancia. Arrumbada la tesis de la distinción entre casos fáciles y casos difíciles aquellos que postulan decisiones basadas en principios por parte del juez- el papel de los principios se hace más amplio y penetrante y emerge siempre con mayor evidencia la idea de cómo el derecho implica constantemente la elección entre diversas interpretaciones de los principios $(249,328)$, de la misma manera la crítica antipositivista se hace más rica y compleja. La interpretación constructiva «consiste en atribuir una determinada intención a un objeto o a un uso, para convertirles en los mejores ejemplos posibles de la categoría o del género al que pertenecen» (54). De tal manera que los desacuerdos sobre los fundamentos del derecho se presentan como desacuerdos relativos al valor moral del derecho en cuanto práctica social. El «interpretivismo» de DWORKIN ${ }^{12}$, es decir, la tesis que el concepto doctrinal del derecho funciona como un concepto interpretativo, al menos en comunidades políticas complejas, y que como tal requiere que las personas compartan una praxis, cuya interpretación es por otra parte muy probablemente controvertida, estimuló un debate muy rico, que tuvo entre sus protagonistas estudiosos del calibre de HART, RAZ y FINNIS ${ }^{13}$.

La teoría interpretativista de DwORKIN, al afirmar que la práctica jurídica es por naturaleza propia interpretativa y desarrollando por consiguiente su perspectiva desde el punto de vista de aquel sujeto activo que investiga y administra el derecho en casos de conflicto, da a su Hércules, que no necesita de un método para distinguir los casos fáciles de los difíciles, una tarea sobrehumana, el moverse en la continua búsqueda de la respuesta justa, pero con una intuición muy simple, pero profunda, reconociendo a cada componente de la comunidad jurídica la posibilidad de involucrarse en argumentaciones morales y políticas con la finalidad de determinar la justificación correcta de la práctica jurídica. Su teoría del derecho parte de una comprensión de los problema de la práctica jurídica en su conjunto (HART, 2002: 186), que se presenta como una práctica argumentativa, dentro de la cual los participantes tienen diferentes pretensiones sobre lo que el derecho exige y defienden tales pretensiones ofreciendo razones para su sostenimiento. El intérprete ideal llega a un equilibrio entre las diversas prácticas interpretativas, teniendo que orientarse no solo sobre la base de un pasado vinculante, sino también atendiendo los estándares reconocidos por el derecho. Visión esta, en la que resultan difícilmente contestables, por lo menos, dos aspectos: por una parte que la implicación del sujeto que entra en juego en la práctica jurisdiccional no puede prescindir de una preliminar mirada hermenéutica ${ }^{14}$, y que tal sujeto no puede prescindir de una interpretación holística, que sitúa el caso individual dentro de un ordenamiento entendido como conjunto integrado por principios y reglas, según una jerarquía de coherencia redefinida de caso en caso y argumentativamente recalibrada. El punto fundamental de contraste con la teoría general de HART, y con el carácter descriptivo del derecho que esta reivindicaba, está precisamente en el hecho de que el derecho, según la opinión de DwORKIN (210b: 154), no viene descrito de manera general y desde el exterior ( $c f r$ RAZ, 1979: 153), como se da en la perspectiva de un estudioso que no

12 Vid. en particular DwORKIN (2010b: 13-15; 1989: 168 y ss).

13 Entre las diversas voces de este debate Mitchell (1983); COHEN (1983); HART (2002); RAZ (1986); FinNis (1992); COLEMAN (2001). Una historia del debate suscitado por los primeros cinco capítulos de Taking Rights Seriously viene trazada por el mismo DwORKIN (2010b: 204-242).

14 Así también Habermas (2011: 157). 
se involucra en los casos jurídicos, sino desde el interior, desde el punto de vista de un participante activo en las disputas jurídicas ${ }^{15}$. La argumentación jurídica se convierte así en una argumentación típica y fundamentalmente moral, de tal manera que los juristas deben decidir qué conjunto de principios en liza suministra la mejor justificación de la práctica jurídica en su conjunto (158).

\section{EL DERECHO COMO INTEGRIDAD}

Hay que subrayar especialmente - lo he hecho ya en mi precedente ensayo (ZACCARIA, 1998) - como estas conclusiones se acercan sensiblemente y demuestran una interesante consonancia y convergencia con las tesis de Josef ESSER: lo que sobretodo diferencia a los dos estudiosos es el diferente contexto jurídico de fondo de sus teorías, europeo-continental en el caso de ESSER, explícitamente estadounidense en el caso de DWORKIN. Se pueden encontrar, sin embargo, semejanzas sugestivas sobre temas claves, como la pre-comprensión y el control de concordancia y de rectitud material, que en el lenguaje dworkiniano viene expresado con la tesis de las dos dimensiones del fit, en correspondencia con las decisiones legislativas y jurisdiccionales precedentes, y de la justificación, como correspondencia con los principios de la comunidad política. Para confirmar el hecho de que no se puede comprender sin un pre-comprender que es condición positiva, si bien provisional, de conocimiento, pero incluso como confirmación de la circunstancia que la indiscutible conexión entre valores éticos y valores jurídicos exige interpretar el derecho a la luz de una visión más amplia de la justicia. Al representar elementos constitutivos del ordenamiento jurídico, los principios expresan por consiguiente exigencias de justicia, de equidad, de corrección e incorporan argumentos que versan sobre los derechos, que, en sintonía con la tradición cultural anglosajona de signo liberal ${ }^{16}$, se configuran como reivindicaciones de libertad para con el poder constituido (o, incluso, contra él). En cuanto preceptos de optimización que reivindican ser realizados de la manera más amplia posible, los principios son sopesados y el problema de su aplicación es también un problema de equilibrio de valores. Los jueces deben basar sus decisiones en argumentos de political principle (y no de political policy); es decir, deben decidir sobre la base de los derechos de los individuos y no sobre la base de finalidades políticas o sociales colectivas (DwORKIN, 1990b: 8 y ss., 15-16, 36). En este sentido es del todo legítimo definir la postura dworkiniana como una «jurisprudencia de los derechos», aun cuando el concepto de derechos de DwORKIN no es en absoluto independiente de las instituciones. Los derechos se hacen valer esencialmente dentro de una práctica social, dando lugar a un conjunto de normas, de comportamientos y de valoraciones con una proyección de carácter universalista.

La integridad ${ }^{17}$, como noción cardinal de la actividad jurisdiccional y de todo el proceso de positivización jurídica - en cuanto concepto central que opera dentro de la práctica social de tipo interpretativo que es el derecho- demuestra estar de hecho íntimamente ligada a la tutela de los derechos de los ciudadanos (en su alcance contra-

15 Desde este punto de vista las tesis de DWORKIN presentan afinidades con FINNIS (1980: 3 ).

16 En este sentido las posiciones de DWORKIN son asimilables a las de RAWLS (1982), SEN (1984), y MACKIE (1977).

17 Sobre el tema, $c f r$. PASTORE (1993) y SCHiavello (1998). 
mayoritario y anti-utilitarista) y ligada asimismo a la garantía de los peculiares valores de la comunidad liberal ${ }^{18}$. El derecho como integridad asume que los derechos derivan de las decisiones tomadas «no solo cuando son explícitos en estas decisiones, sino también cuando son consecuentes con los principios de moralidad personal y política que las decisiones explícitas presuponen como su justificación» (DwORKIN, 1989: 94, 130) ${ }^{19}$. La concepción del derecho como integridad requiere que la interpretación de los materiales normativos se realice a la luz de los principios fundamentales de la comunidad, cuyo reconocimiento institucional es realizado por el juez. La coherencia en cuanto criterio, justificativo de la decisión judicial, tiene como punto de referencia el set de principios que permite a los jueces - teniendo en cuenta la exigencia de conciliación de los principios- hallar la solución que sea coherente con las ideas de moralidad política enraizadas en la estructura general de la misma práctica jurídica (129, 207 y ss., 214, 317). La exigencia de la coherencia ordinamental implica por consiguiente el reenvío a los principios - asumidos como tejido conectivo del corpus jurídico- que se refieren constitutivamente a las premisas axiológicas propias del Estado de derecho y exigen la realización del ideal jurídico incorporado por este último (371 y ss., 375 y ss.; cfr. también ALEXY, 1997: 74 y ss., 80-81). Desde esta perspectiva, el derecho se caracteriza como interpretación de datos de partida a la luz de los principios institucionales, que constituyen la medida para valorarlos, reconstruyendo las líneas de tendencia y las interrelaciones ordinamentales. También aquí se explica su «fuerza gravitacional» (DWORKIN, 2010a: 211 y ss.), que expresa una potencialidad normogenética y les hace operar como guidelines de decisiones, que de otro modo estarían al arbitrio de sus autores. Podemos concluir que en la base de la teoría dworkiniana de los principios se da, en consecuencia, la idea de que la experiencia jurídica sustenta un diseño coherente y unitario, cognoscible en vía sistemática. Los principios, de hecho, nos retraen a la idea de una matriz de la que el derecho es expresión, pero impregnan también la imagen de una realización armoniosa de sus partes, ya que está inspirada en corrientes y valores unitarios ${ }^{20}$. Una concepción, la de DwORKIN, que tiene que saldar cuentas con la realidad contemporánea de un derecho cada vez más heterogéneo y estratificado, compuesto por bloques de materiales normativos guiados por lógicas diferentes, cuando no incluso incompatibles.

De este modo DwORKIN basa su filosofía del derecho sobre el fundamento más amplio de un individualismo ético, sobre la generalización de un deber moral al realizar el valor objetivo de la existencia, tanto individual como social. El individualismo ético propuesto por DwORKIN, cuando afronta en Sovering Virtue el tema de la posible contradicción entre derechos del individuo e interés general, se basa ya sea sobre la igualdad, como sobre la responsabilidad personal respecto al tipo de vida y a los medios elegidos para la propia realización.

La responsabilidad que tenemos en lo que se refiere a nuestra vida está en la base de la responsabilidad contemporánea para apoyar a los demás en la realización de

18 Acerca de este tema $c f r$. SCHIAVELLO (1998: 10 y ss., 251 y ss.) y DwORKIN (1990a: 1-33).

19 Como ya se ha hecho notar (Postema, 1987: 294) la teoría del juicio jurisdiccional elaborada en Law' Empire incorpora la rights thesis de Taking Rights Seriously.

${ }^{20}$ De aquí proviene la crítica a la teoría de DwORKIN de mirar optimistamente y de manera utópica la realidad jurídica a través de «filtros rosas»; $c f r$. HuTCHINSON (1987: 653). 
este mismo deber. Más que un intento por perfeccionar desde dentro el utilitarismocomo querría HART (1979: 841 y ss.) — creando en torno al individuo una barrera apta para defenderlo en el posible conflicto con el bienestar general (Yowell, 2007: 130 y ss.), la jurisprudencia de DwORKIN parece inspirarse en el principio de derivación aristotélica de igual consideración y respeto.

\section{JUSTICE IN ROBES: LA VALORIZACIÓN DE LA ARGUMENTACIÓN MORAL}

Justice in Robes ${ }^{21}$ constituye un punto sin retorno en la perspectiva dworkiniana, en la que las consideraciones de carácter moral son parte integrante de los criterios para determinar el derecho válido. La tenaz y constante propuesta de su argumento crítico «contra HART»: «según mi concepción, la argumentación jurídica es una argumentación típica y fundamentalmente moral» (DWORKIN, 2010b: 158), mientras en la teoría hartiana «la existencia y el contenido del derecho pueden ser reconocidos [...] sin referencia a la moral» (157) no podría ser más clara y nítida. Las consideraciones morales son relevantes siempre, y no solo cuando son reconocidas por las fuentes del derecho como la legislación o las decisiones judiciales, como ocurre en cambio en el caso de HART. Se podría decir, en otras palabras, que el internal point of view, como guía para valorar y juzgar tanto el comportamiento propio como el de los demás, se convierte aquí en una moral perspective (GREEN, 2005: 1955), desde el momento que una descripción, una teoría empírica o descriptiva constituyen obviamente en sí mismas una empresa en algún sentido normativa (DWORKIN, 2010b: 180) y son en consecuencia auto-contradictorias.

La perspectiva de HART, como por otra parte la de gran parte de la filosofía especializada contemporánea, se puede críticamente definir como archimedea, precisamente porque su análisis del concepto de derecho es una actividad de mera descripción de una práctica jurídica, que se quiere mantener programáticamente alejada de los esfuerzos más concretos de los juristas y jueces a la hora de determinar lo que es legal y lo que es ilegal. A esta perspectiva, a la jurisprudence descriptiva, DwORKIN (185-186) contrapone la teoría general elaborada en El imperio del derecho, es decir una teoría filosófica que parte de una comprensión de los problemas integrantes de la práctica jurídica y que incluye en su interior la legalidad como un ideal interpretativo.

El foco del intenso debate con HART está representado, una vez más, y sobre la estela del Imperio del derecho, por el llamado «semantic sting», que ya en el primer capítulo de aquella obra nuclear de DwORKIN (1989: 35 y ss.) había constituido el eje fundamental de su crítica a las teorías semánticas ${ }^{22}$. De acuerdo con la tesis dworkiniana, las teorías semánticas, y entre ellas in primis la de HART, no son capaces de explicar dentro de la praxis jurídica lo que constituye un elemento estructural, es decir la discrepancia, ya que consideran que los juristas comparten tests no controvertidos suministrados por el significado convencional de la palabra derecho. El desacuerdo podría entonces centrarse solo sobre cuestiones empíricas o limitarse a los llamados

\footnotetext{
21 Sobre este libro de DwORKIN hay que ver la nota de GREEN (2007).

22 Sobre el semantic sting, $c f r$. la crítica de RAZ (1998).
} 
«casos de penumbra», pero nunca conferir la misma interpretación de la práctica del derecho. En definitiva, el desafío lanzado por DWORKIN a HART concierne precisamente a la explicación e inclusión del elemento del desacuerdo dentro de la práctica jurídica y la sutileza de su argumento antipositivista plantea la duda de que los juristas que niegan la existencia de discrepancias teóricas dentro del derecho se entregan de manera rutinaria a argumentaciones incoherentes sin ni siquiera darse cuenta de su error $^{23}$.

Es bien sabido que en el Postscript HART , que consideraba cruciales las objeciones de DwORKIN, hasta el punto de hacer de ellas el objeto principal de su apéndice, negó con gran vigor, aunque no siempre felizmente, esta interpretación de DwORKIN, que se exponía a poner en crisis profunda su proyecto de descripción del derecho, y afirmó que su tesis había sido profundamente tergiversada corroborando que la suya pretendía ser una teoría no normativa o valorativa del derecho, sino más bien una teoría empírica o descriptiva, de la cual por otra parte, objeta DWORKIN (210b: 182), ni HART ni sus seguidores han iniciado siquiera los amplísimos estudios empíricos que serían necesarios para fundamentarla.

Es verdad que la crítica dworkiniana es a su vez susceptible de objeciones, desde el momento que pretende formular una crítica general al modelo de HART en términos de filosofía del lenguaje, y más exactamente de crítica a las teorías semánticas y al convencionalismo metasemántico de gran parte de la filosofía contemporánea. El error de DWORKIN — ha sostenido GREEN (2007: 1498; 2003, 1897 y ss.) — está en asumir que la práctica jurídica es equivalente a la práctica lingüística que usa el concepto de derecho. Pero aun prescindiendo de similares consideraciones, la objeción a la tesis por la que los componentes de una comunidad pueden emitir juicios jurídicos solo si comparten tests no controvertidos de validez jurídica, capaces de agotar los fundamentos sobre la base de los cuales tales juicios están legalmente justificados, se mantiene firme con una consistencia que permite captar la estrechez del modelo hartiano en el momento en que niega que el concepto de derecho se use dentro de una práctica interpretativa en la que su correcta aplicación está determinada no por reglas semánticas generales, sino más bien por la teoría que ofrece la mejor interpretación.

Las dificultades ligadas a la perspectiva formalista sobre el tema de la interpretación y de la relación entre derecho y valores en sede aplicativa se muestran progresivamente evidentes en el iuspositivismo post-hartiano. Partiendo del amplio debate que ha surgido en torno al Postscript en la segunda edición de The Concept of Law el iuspositivismo se ha ido de hecho articulándo en una doble dirección, el del «iuspositivismo exclusivo» y el del «iuspositivismo inclusivo» (o soft positivism). Mientras el iuspositivismo exclusivo (RAZ, 1979) se mantiene fiel a la línea más tradicional de la separación entre derecho y moral, el iuspositivismo inclusivo (MORESO, 2003; WALDRON, 1992; WALUCHOW, 1994), reconociendo implícitamente la validez de algunos elementos de la crítica dworkiniana, se empeña en formular una concepción del derecho (y de la interpretación jurídica) que, precisamente, incluya los valores, de manera compatible con la fidelidad al tema de la distinción entre derecho y moral (SCHIAVELLO, 2004).

23 SHAPIRO sostiene la competibilidad de la crítica dworkiniana con el mantenimiento de una perspectiva iuspositivista (2007: 42). 
El iuspositivismo inclusivo propone, con buen criterio, una reformulación de la regla de reconocimiento hartiana de manera que incluya la referencia a elementos morales, con incidencias importantes bajo el perfil de la relación entre derecho y moral respecto a la definición del concepto de derecho, de la validez de las normas y de la cuestión de la discrecionalidad judicial. En base al iuspositivismo inclusivo, estándares de moralidad política pueden figurar de distintas maneras en los intentos de determinar la existencia, el contenido y el significado del derecho válido. Como oportunamente ha llamado la atención Vittorio VILLA (2000), para distinguirse nítidamente y de manera verdaderamente fértil del exclusive positivism, el positivismo inclusivo debería sin embargo tener éxito no tanto en reconocer la existencia, en el derecho positivo, de reenvíos a la dimensión moral, cuanto más bien subrayar la fluidez de la relación entre elementos jurídicos y elementos morales dentro de las normas jurídicas, o al menos de ciertas normas jurídicas, como por ejemplo en el ámbito del derecho constitucional. En cualquier caso, tanto después de la publicación del Postscript de HART, como después de la nueva discusión de sus tesis por parte de DwORKIN en La Justicia en toga, la controversia HART-DwORKIN no ha cesado de interesar a la comunidad científica de los teóricos del derecho - signo elocuente del carácter duradero de los temas suscitados en ella, que aluden a una de las cuestiones más profundas de la filosofía del derecho, la referida a la relación entre derecho y moral (cfr. SHAFIRO, 2007: 5) -lo que originó una gran producción de numerosas contribuciones, no siempre útiles sin embargo, dada la frecuente diversidad y el desacuerdo entre los puntos de partida de los diversos autores- ${ }^{24}$. Pero en cualquier caso sobre la querella HART-DwORKIN se han formado distintas generaciones de estudiantes de las law schools americanas y la importancia fundamental de esta discusión intelectual que ha involucrado a fondo dos concepciones distintas del derecho, permanece la misma todavía hoy cuarenta años después de su primera formulación.

\section{DEL PLURALISMO DE LOS VALORES AL HOLISMO DEL VALOR}

Justice for Hedgehogs ${ }^{25}$, libro brillante y de gran ambición, representa verdaderamente en su enfoque sistemático - pero refractario a las divisiones disciplinarias - el culmen y la articulación conclusiva de toda la teoría del derecho dworkiniano recogiendo en el fondo unitariamente los resultados de décadas de reflexiones. De las normas a los principios, de la confrontación crítica con el iuspositivismo y con el paradigma hartiano a la pregunta fundamental sobre la naturaleza del derecho, la «ascensión filosófica» del largo recorrido de investigación de DWORKIN ${ }^{26}$ exige finalmente una visión más amplia y llega a plantearse la cuestión fundamental de cuáles son las verdades en ética y en moral. Sin fingimientos, DwORKIN (2013a: 457) se hace cargo de la circunstancia de que un cuadro teórico que presente los dos sistemas de la moral y del derecho como sistemas que están separados, si bien vinculados por momentos específicos de conexión, se expone inevitablemente a lo que él define como un «defecto fatal»: aquel por el que no

${ }^{24}$ Entre las muchas voces del debate hay que tener en cuenta Himma (2000: 2002); MARMOR (2005); KRAMER (2000: 2002); SHAPIRO (2007); SMITH (2009: 2010); LEITER (2003).

25 Ya el título hace referencia explícita al famoso ensayo de Isaiah BERLIN (1978). Una importante colección de ensayos sobre este volumen fundamental de DWORKIN está recogida en un número especial de la Boston University Law Reviev (VVAA, 2010).

26 Es el mismo DwORKIN (2013b: 6) el que habla de «process of philosophical ascent». 
existe ningún punto de vista neutral a partir del cual examinar las conexiones entre los dos sistemas supuestamente separados. Ante el peligro de ser expuesto a la controversia, todo análisis del concepto de derecho tiene, dentro de esta perspectiva, que asumir desde el principio la idea de una estrecha conexión entre derecho y moral, hasta concluir que el derecho tiene que ser tratado como una parte de la moral política (459), y por consiguiente como un momento específico de la teoría general de la ética. La existencia y el contenido del derecho positivo son entonces en un último análisis dirigidos y gobernados por la existencia y el contenido de la ley moral. La justicia deriva de la dignidad y aspira a la dignidad (478). Esto significa apartar toda suerte de escepticismo y asumir sin reservas la dimensión del cognitivismo ético, creyendo en la realidad objetiva y en la unidad del valor (474 y ss.) ${ }^{27}$, que no entra en conflicto con el hecho de que deba adaptarse a las «situaciones humanas» y pueda ser objeto de práctica de equilibrio pero en cualquier caso no excluye obviamente por sí mismo la tolerancia y el respeto recíproco. Desde el punto de vista kantiano es propio de nuestra dignidad respetar la dignidad de los demás $^{28}$. Es posible discutir críticamente de valores solo reconociéndoles un valor y un significado objetivos. Nuestras convicciones morales y nuestros valores forman un conjunto coherente, en el que cada valor particular está capacitado para reforzar y sostener otro valor (DWORKIN, 2007: 10-11). DwORKIN (2013a: 13) se da perfectamente cuenta que su tesis general —el valor es una cosa importantísima, y el análisis filosófico del valor debe ser comprometido e impositivo de normas y no descomprometido y neutral - es impopular; especialmente en el contexto anglo-americano donde prevalecen los «zorros» no cognitivistas, mientras los «erizos» se arriesgan a ser tachados de ingenuos o charlatanes. En una época de radical secularización y de gran pluralismo de los valores y las creencias, el holismo del valor de DwORKIN es andar a contracorriente respecto al espíritu de la época y representa verdaderamente la tercera y última fase y al mismo tiempo la articulación concluyente de su pensamiento. A pesar de esto, mantiene muy firme, incluso con intransigencia, la tesis fundamental de que el debate sobre la ética y sobre la política no puede renunciar a un compromiso de fondo con la verdad y con la justicia y en dialogo polémico con la reflexión metaética, y en particular con las tesis de Isaiah BERLIN, ratifica la posibilidad de edificar una perspectiva unitaria de los distintos valores morales que se confrontan en las sociedades pluralistas de nuestro tiempo, desde el momento de que tales valores, lejos de basarse sobre hechos, se fundamentan sobre interpretaciones que pueden ser considerados compatibles entre ellos y coherentes en los diversos ámbitos de la ética y de la política. También el razonamiento moral es un razonamiento interpretativo, capaz de implicarse en la búsqueda de una composición armoniosa en equilibrio de las diversas interpretaciones (FALLON, 2010, 539 y ss.).

Las ambiciones teóricas de Justice for Hedgehogs intentan poner en evidencia como modalidades comunes del proceso interpretativo los distintos ámbitos de la ética y de la política: la teoría interpretativa ilumina acuerdos y conflictos entre los críticos en todos estos campos y sitúa por consiguiente a la interpretación en el centro del ámbito del valor, de cómo responder a preguntas fundamentales, como aquellas que plantean qué significa la buena vida (living well) y como se debe tratar al otro (living a good life). La interpre-

27 Para una crítica cfr. SOLum (2010: 557 y ss.). Posición afín es la de AleXY (2012).

${ }_{28}$ Una defensa de la vinculación entre el pensamiento de DWORKIN y la filosofía kantiana la encontramos en ALLARD (2001). 
tación se convierte en una práctica general de tipo normativo, que permite comprender una gran variedad de prácticas humanas, a excepción de las científicas, como instancias interpretativas. La práctica interpretativa es esencial porque nuestros valores generales y abstractos deben ser aplicados con el fin de determinar lo que se debería hacer en los singulares casos concretos. El acto interpretativo resulta fundamental para la vida moral.

Hay también un riesgo en el proyecto dworkiniano de Justice Hedgehogs: el de difuminar el derecho transformándolo como apéndice de la moral (y no por casualidad aquí el derecho se convierte en objeto de un focus específico solo en un breve capítulo de la obra, mientras en Law's Empire se presentaba una teoría del derecho mucho más desarrollada y sistemática) ${ }^{29}$. Sin embargo, el derecho suministra por su parte a DwORKIN la clave de bóveda para edificar una teoría moral de carácter orgánico: en consecuencia, por un lado el jurista conoce el derecho a través de la interpretación, por otra parte la interpretación se convierte en la base para construir una teoría ética que vuelve a englobar el derecho A partir de las cuestiones y de las demandas de la práctica concreta del derecho se llega a los temas más arduos y generales de la indagación filosófica (VECA, 2013:4).

La peculiaridad de la jurisprudence dworkiniana y sobre todo sus resultados últimos en el tema de las relaciones entre derecho, moral y política tienen el riesgo - por otra parte- de un desequilibrio progresivo a favor de los factores de tipo moral (PASTORE, 1993: 77-78), o sea de redimensionar excesivamente la función de los factores jurídicos, la relación «interna» que se debería mantener entre el material jurídico y la elaboración de la regla del caso (VIOLA, 1990: 123-124). Sin embargo se afirma que, a los fines de la adecuación a los estándares normativos ético-políticos la reconstrucción sistemática del material jurídico y el mismo derecho positivo ofrecen una aportación que no hay que infravalorar. El reconocimiento del carácter autoritario y autónomo del derecho no es si embargo incompatible con su inclusión en una visión unitaria y comprensiva que reconozca la presencia y el enraizamiento de la práctica interpretativa en la moral.

\section{CONCLUSIÓN}

Si bien los escritos de DwORKIN, fruto de un generoso compromiso intelectual en muchos frentes, han sido objeto de vivas críticas y de encendidas refutaciones, su impacto sobre la filosofía del derecho contemporánea ha sido en cualquier caso muy relevante. A partir de HART y en lo sucesivo, no se ha podido dejar de debatir las ideas de DWORKIN y de dedicarles ensayos y conferencias. En consecuencia, podemos tranquilamente reconocer que ha cambiado profundamente el panorama de la teoría del derecho contemporánea.

Como hemos visto, sus escritos filosófico-jurídicos, contribuyendo de manera decisiva a abandonar definitivamente la concepción vetero-positivista del derecho, han reorientado completa y profundamente el debate sobre el positivismo jurídico y sobre el iusnaturalismo (OlLERO TASSARA, 2014). De hecho estas dos concepciones han sido de algún modo inducidas a revisar sus posiciones más rígidas y a abrirse a razones distintas de las que anteriormente sostuvieron.

\footnotetext{
29 Sobre este punto $c f r$. Mc. Clain (2010: 864 y ss.).
} 
Extremamente interesante es la importancia por la que, considerada en su conjunto, la jurisprudence dworkiniana, según la cual el saber jurídico nace en la praxis y para la praxis (BITTNER, 1988), reivindica la función crucial de la actividad interpretativa en el proceso de constitución jurídica, llegando — por vías originales e inspiradas en el análisis del modelo institucional angloamericano- a conclusiones convergentes con las sostenidas por la perspectiva de la hermenéutica jurídica del ámbito continental. En particular lo que parece conciliar las dos perspectivas - la teoría del derecho de DWORKIN por un lado, y la hermenéutica-jurídica por otro- es el reconocimiento de que la interpretación puede tener éxito pero también fracasar, como asimismo la preocupación de evitar los riesgos de un pragmatismo extemporáneo, que ante la toma de conciencia, de que el núcleo fuerte de la praxis jurídica radica en hallar la solución a los casos concretos que se presenten, confía al decisionismo arbitrario del interprete la solución de los problemas que surgen de la praxis y consecuentemente confía en contener la discrecionalidad entendida en sentido fuerte de los jueces. DWORKIN persigue este objetivo a través de una fuerte vinculación con la theory of adjudication, es decir con una teoría prescriptiva del juicio jurisdiccional explícitamente configurada sobre las características procesales e institucionales del sistema angloamericano, mientras la hermenéutica jurídica continental insiste mayormente, como ocurre en las tradiciones del civil law, en el elemento del método y de los vínculos convencionales que caracterizan al derecho, además de la función del jurista-intérprete en la formación del derecho. Sin embargo el objetivo, en términos de teoría del razonamiento jurídico, es común, y consiste en vincular al intérprete de manera estructural, aferrando su praxis a exigencias de coherencia con el conjunto del derecho o bien con los principios de moralidad que están en la base de la convivencia civil.

\section{BIBLIOGRAFÍA}

AleXY, R., 1997: Concetto e validità del diritto (1985), Torino: Einaudi.

— 2000: «On the Structure of Legal Principles», Ratio Juris, 13: 294-304.

- 2012: Teoria dei diritti fondamentali (1986), Bologna: Il Mulino.

AllarD, J., 2001: Dworkin et Kant. Reflexions sur le judgment, Editions de l'Université de Bruxelles.

Atienza, M., y J. Ruiz MAnero, 1993: «Tre approcci ai principi di diritto», Analisi e diritto, 9-29.

- 1996: Las piezas del Derecho. Teoría de los enunciados jurídicos, Barcelona: Ariel.

BERLIN, I., 1978: «The Hedgehog and the Fox», Russian Thinkers, 22: 22-81.

BITTNER, C., 1988: Recht als interpretative Praxis zu Ronald Dworkins allgemeiner Theories des Rechts, Berlin: Duncker und Humbolt.

Cohen, M., 1984: Ronald Dworkin and Contemporary Jurisprudence, London: Duckworth.

Coleman, J., 2001: Hart's Postscript, Oxford: Oxford U. P.

Comanducci, P., 1997: «Principi giuridici e indeterminazione del diritto», Analisi e diritto, 55-68.

Duarte D'Almeida, L.; Edwards, J., y Dolcetti, A. (eds.), 2013: Reading H.L. Hart's «The Concept of Law», Oxford: Hart Publishing.

DwORKIN, R., 1975: «Hard Cases», Harvard Law Review, 88: 1057-1109.

- 1989: L'impero del diritto (1986), Milano: Il Saggiatore.

- 1990a: Questioni di principio (1985), Milano: Il Saggiatore. 
— 1990b: «La comunità liberale», Teoria politica, 1: 1: 33.

- 1996: The Moral Reading of American Constitution, Cambridge (Mass.): Harvard University Press.

- 2000: The Theory and Practice of Equality, Cambridge-London: Harvard University Press.

- 2007: La democrazia possibile: principi per un nuovo dibattito politico (2006), Milano: Feltrinelli.

- 2010a: I diritti presi sul serio (1977), Bologna: Il Mulino.

- 2010b: La giustizia in toga, Roma-Bari: Laterza.

- 2013a: Giustizia per i ricci (2006), Milano: Feltrinelli.

- 2013b: Religione senza Dio (2013), Milano: Feltrinelli.

— 2013c: «Legal Theory from the Inside Out», Notizie di Politeia, XXIX: 109 ss.

EndicotT, T., 2013: La generalità del diritto, Modena: Mucchi.

FALLON, R. H., 2010: «Is Moral Reasoning Conceptual Interpretation?», Boston University Law Review, 90: 535-550.

FINNIS, J., 1982: Natural Law and Natural Rights, Oxford: Clarendon Press.

- 1992: «Natural Law and Legal Reasoning», en R. GEORGE (ed.), Natural Law Theory, 134157, Oxford: Clarendon Press.

GreEn, M. S., 2003: «Dworkin's Fallacy, Or What the Philosophy of Language Can't Teach Us About the Law», Virginia Law Review, 89: 1897-1952.

- 2005: «Legal Realism as Theory of Law», William and Mary Law Review, 6: 1917-200.

— 2007: «Dworkin v. the Philosophers: A Review Essay on Justice in Robes», University of Illinois Law Review, 5: 1477-1527.

HABERMAS, J., 2011: «Ronald Dworkin. Un solitario nella cerchia degli studiosi di diritto», en id., Il ruolo dell'intellettuale e la causa dell'Europa, Roma-Bari: Laterza.

Hart, H. L. A., 1979: «Bentham Between Utility and Rights», Columbia Law Review, 79: 828-846.

- 2002: Il concetto di diritto (1994), 2. ${ }^{\text {a }}$ ed., Torino: Einaudi.

Hershovitz, S., 2006: Exploring Law's Empire. The Jurisprudence of Ronald Dworkin, Oxford: Oxford University Press.

Himma, K. E., 2000: «H. L. A. Hart and the Practical Difference Thesis», Legal Theory, 6: 1-43.

— 2002: «Ambiguously Stung: Dworkin's Semantic Sting Reconfigured», Legal Theory, 2: 145-183.

Hutchinson, A. C., 1987: «Indiana Dworkin and Law's Empire», The Yale Law Journal, 96: 637-655.

Kramer, M., 2000a: «How Morality Can enter the Law», Legal Theory, 6: 83-108.

- 2000b: «Throwing Light on the Role that Moral Principle Play in the Law», Legal Theory, 8: $115-143$.

LeITER, B., 2005: «Beyond the Hart-Dworkin Debate: The Methodology Problem in Jurisprudence», University of Texas Law, Public Law Research Paper, 34.

MACKIE, J., 1977: Ethics: Inventing Right and Wrong, Harmondsworth: Penguin Books.

- 1984: «The Third Theory of Law», en M. COHEN (ed.), Ronald Dworkin and Contemporary Jurisprudence, Totowa (N. J.): M. Cohen, Rowman \& Allaheld, 172-181.

MARMOR, A., 2006: Interpretation and Legal Theory, Oxford-Portland: Hart Publishing.

MCLAIN, L., 2010: «Justice and Elegance for Hedgehogs - In Life, Law, and Literature», Boston University Law Review, 90: 864-902.

Mengoni, L., 1996: «L'argomentazione nel diritto costituzionale», en id., Ermeneutica e dogmatica giuridica. Saggi, Milano: Giuffré, 115-140.

MindA, G., 2002: Teorie postmoderne del diritto, Bologna: Il Mulino.

MitCHeLl, T., 1983: The Politics of Interpretation, Chicago-London: University of Chicago Press. 
MoreSO, J. J., 2003: «El reino de los derechos y la objetividad de la moral», en E. DiciotTi (ed.), Diritti umani ed oggettività della morale, Siena: Dipartimento di scienze storiche, giuridiche, politiche e sociali, 9-40.

PASTORE, B. 1985: I principi ritrovati, Palermo: Ila Palma.

- 1993: «Integrità, tradizione, interpretazione», Rivista internazionale di filosofia del diritto, 70: 43-78.

PINO, G., 2010: Diritti e interpretazione. Il ragionamento giuridico nello stato costituzionale, Bologna: Il Mulino.

Pintore, A., 1982: Norme e principi. Una critica a Dworkin, Milano: Giuffré.

Postema, G., 1987: «"Protestant” Interpretation and Social Practices», Law and Philosophy, 6: 283-319.

RAWLS, J., 1982: Una teoria della giustizia (1971), Milano: Feltrinelli.

RAZ, J., 1978: «Professor Dworkin's Theory of rights», Political Studies, 26: 123-137.

- 1979: The Authority of Law, Oxford: Oxford University Press.

- 1986: Dworkin: «A New Link in the Chain», California Law Review, 74: 1103-1119.

— 2003: «Two Views of the nature of law: A Partial Comparison», Legal Theory, 9: 249-282.

RIPSTEIN, A., 2007: Ronald Dworkin, Cambridge: Cambridge University Press.

SCHiavello, A., 1998: Diritto come integrità: incubo o nobile sogno? Saggio su Ronald Dworkin, Torino: Giappichelli.

- 2004: Il positivismo giuridico dopo Herbert L. A. Hart, Torino, Giappichelli.

SEN, A., 1984: «Rights and capabilities», en id., Resources, Values and Development, Oxford: Blackwell, 307-324.

SHAPIRO, S., 2007: «The "Hart-Dworkin” Debate: A Short Guide for the Perplexes», University of Michigan Law School, Working paper, 77.

SMITH, D., 2009: «Has Raz Drawn the Semantic Sting?», Law and Philosophy, 28: 291- 325.

- 2010: «Theoretical Disagreement and the Semantic Sting», Oxford Journal of Legal Studies, 30: 635-661.

Solum, L., 2010: «The Unity of Interpretation», Boston University Law Review, 90: 551-578.

VeCA, S., 2013: «La lezione di Ronald Dworkin», Notizie di Politeia, 109: 3-5.

VILLA, V., 2000: «Alcune chiarificazioni concettuali sulla nozione di inclusive positivism», Analisi $e$ diritto, 255-288.

ViOLA, F., 1990: Il diritto come pratica sociale, Milano: Jaca Book.

VVAA, 2010: «Justice for Hedgehogs: A Conference on Ronald Dworkin's Forthcoming Book», Boston University Law Review, 2: 465-1086.

WALDRON, J., 1992: «The Irrelevance of Moral Objectivity», en R. P. GEORGE (ed.), Natural Law Theory. Contemporary Essays, Oxford: Oxford University Press, 158-187.

Waluchow, J. W., 1994: Inclusive Legal Positivism, Oxford: Clarendon Press.

Yowell, P., 2007: «A critical examination of Dworkin's theory of rights», American Journal of Jurisprudence, 52: 93-138.

ZACCARIA, G., 1996: Questioni di interpretazione, Padova: Cedam.

- 1998: «Precomprensione, principi e diritti nel pensiero di Josef Esser. Un confronto con Ronald Dworkin», Ragion pratica, 11: 135:152.

ZagrebelsKy, G., 1992: Il diritto mite. Legge, diritti, giustizia, Torino: Einaudi. 